\title{
Psicopatía, violencia y criminalidad: un análisis psicológico-forense, psiquiátrico-legal y criminológico (Parte I)
}

\author{
Psychopathy, violence and crime: a psychological-forensic, \\ psychiatric-legal and criminological analysis (Part I)
}

\section{Resumen}

Tras más de 200 años de historia, el concepto de "psicopatía" ha venido experimentando una auténtica y variopinta metamorfosis terminológica. Exceptuando aquellos períodos del Medievo en los que se creía que la psicopatía y cualquier otra conducta y personalidad "anormales" eran bien de origen demoníaco, bien de corte estrictamente hereditarista-biologicista-fisionomista-antropométrico, el resto de enfoques teórico-investigadores han arrojado sobrada luz que, a día de hoy, y tras más de 200 años de historia amarga y controvertida sobre el término, nos permiten haber llegado a un consenso generalizado entre los estudiosos de la psicopatía desde el punto de vista clínico-forense y criminológico.

Este primer trabajo o Parte I es una revisión en la que se pretende ahondar en los polémicos y muchas veces incomprobables orígenes del término "psicopatía". Por otro lado, y tras intensas investigaciones empíricas realizadas desde los años 70 y centradas fundamentalmente en la población penitenciaria, modernamente se ha comenzado a hablar, de manera distintiva, tanto de los psicópatas criminales como de los psicópatas integrados, habiéndose hallado que la diferencia principal entre ambos tipos de psicópatas estriba, sencillamente, en la concreta comisión de delitos, puesto que la estructura básica de personalidad es prácticamente la misma en ambos tipos de psicópatas. Finalmente, se hace muy necesario subrayar que el carácter antisocial de la personalidad y conducta se observa, por definición, en cualquier tipo de delincuentes, sean éstos o no psicópatas, un carácter antisocial que, por otro lado, no siempre ni necesariamente se observa entre los denominados psicópatas integrados.

Palabras clave: Antisocial. PCL-R. Personalidad psicopática. Psicopatía. Sociopatía.

\section{Abstract}

After more than 200 years of history, the concept of "psychopathy" has been undergoing an authentic and varied terminological metamorphosis. Except for those periods in the Medieval Age in which it was believed that psychopathy and other "abnormal" behaviors and personalities were either from a demonic origin, or from a strictly hereditarianbilogicist-physiognomic-anthropometric origin, the other theoretical-research approaches have cast on light that, on today, and after over 200 years of bitter and controversial history of the term, allow us to have reached a broad consensus among scholars of psychopathy from a clinical-forensic and criminological standpoint.

This first paper or Part I is a review that aims to delve into the controversial and often unverifiable origins of the term "psychopath". On the other hand, after intense empirical research since the 70's and focused primarily on the prison population, modernity it has begun to talk differently about both criminal psychopaths as successful psychopaths, having found that the main difference between the two types of psychopaths lies simply in the concrete crimes, being as the basic structure of personality is almost the same in both types of psychopaths. Finally, it's very necessary to emphasize that the antisocial character of the personality and behavior is observed, by definition, in any criminal, whether or not psychopaths, an antisocial character, on the other hand, which does not always or necessarily seen between the so-called successful psychopaths.

Key words: Antisocial. PCL-R. Psychopathic personality. Psychopathy. Sociopathy.
JM. Pozueco Romero ${ }^{1}$ SL. Romero Guillena² N. Casas Barquero ${ }^{3}$

\footnotetext{
${ }^{1}$ Doctorando en Psicología (Área de Personalidad, Evaluación y Tratamiento Psicológico, Facultad de Educación, Universidad de Extremadura,

UNEx, Badajoz) Experto en Psicología Criminal y Forense (UVEG, UCM).

${ }^{2} \mathrm{FEA}$ en Psiquiatría de la Unidad de Gestión Clínica del Área Hospitalaria (HU Virgen Macarena, Sevilla).

${ }^{3}$ Doctora en Psiquiatría. Profesora del Departamento de Psiquiatría

(Facultad de Medicina, Universidad de Sevilla).
}

Correspondencia: J. Manuel Pozueco Romero. E-mail:

jmpozueco@hotmail.com

Fecha de recepción: 1.JUL.2011

Fecha de aceptación: 26.JUL.2011 


\section{Introducción: las ciencias sociales y de la salud mental ante los psicópatas}

En 1996, el doctor Robert D. Hare ${ }^{1}$ escribió un artículo de revisión titulado Psychopathy: A clinical construct whose time has come ("Psicopatía: Un constructo clínico para los tiempos que vienen") que fue publicado en la prestigiosa revista Criminal Justice and Behavior. Sin duda alguna, este artículo de revisión puso magistralmente de manifiesto la situación actual en la que se encontraba la psicopatía, tanto a nivel teórico como a nivel de investigación. Se trata de uno de los artículos más citados de entre los incontables trabajos que ha publicado Hare y el cual ha servido de referencia para los diversos investigadores de todo el mundo, ya que incluso ha servido de base para la elaboración de posteriores artículos de revisión y capítulos de libro en castellano.

Recientemente, en otro espléndido artículo de revisión español que vuelve a rememorar el del doctor Robert Hare, Torrubia y Cuquerella ${ }^{2}$ han puesto de manifiesto una realidad psicosocial que es la que interesa traer aquí a colación: "la psicopatía es una de las entidades clínicas más controvertidas, y ello se debe a diversos elementos de confusión que se sitúan en dos planos distintos: el conceptual y el terminológico" (p. 26).

Las fuentes históricas que nos pueden traer a la memoria ejemplos de psicópatas pueden ser tantas como de sí dé la imaginación de cada historiador. Sin embargo, y a pesar de que podemos encontrar incluso referencias bíblicas e históricas de algunos personajes que más o menos emulan al psicópata ${ }^{3}$, lo cierto es que la psicopatía no comenzó a tomar forma como un constructo clínico con entidad propia hasta principios de 1940, fundamentalmente con la influyente obra del psiquiatra norteamericano Hervey Milton Cleckley: The Mask of Sanity ${ }^{4}$.

En la presente revisión se realiza un amplio y detallado recorrido histórico sobre el concepto de psicopatía desde las Ciencias Sociales y de la Salud Mental, atravesando por varias de las disciplinas y subdisciplinas incardinadas en ambas y que se han preocupado por el estudio de la psicopatía tanto a nivel teórico como empírico. Veremos que a lo largo de más de dos siglos de historia del término, la psicopatía se ha venido denominando de múltiples maneras; en un principio se intuía que existían personas como las que actualmente calificamos de psicópatas, sólo que el término más adecuado no cuajaba con los enfoques imperantes y que estaban en constante disputa y excesivamente centrados en su etimología.

\section{La psiquiatría médica: ¿locos pero cuerdos?}

La conceptualización que en este período histórico y a través de la antigua perspectiva médico-psiquiátrica se tenía con respecto a la psicopatía es, semánticamente hablando, desconcertante y desorientadora: ¿cómo era posible tratar de concebir a personas que, por un lado, parecían estar "locas" pero, por otro lado, realmente cuerdas?

Parece ser que en aquellos tiempos todo era posible, sobre todo a nivel teórico-conceptual. Aunque el trasfondo de lo que los autores de este período pretendían transmitir se puede llegar a entender actualmente, lo cierto es que ese tipo de concepciones desde una perspectiva estrictamente psicopatológica le ha hecho un daño tremendo a la psicopatía, la cual, incluso en los tiempos presentes, sigue teniendo muy mala prensa.

\section{La manie sans délire (manía/locura sin delirio) y la moral insanity (locura moral)}

Conocida generalmente en aquellos tiempos como la folié raisonnante (locura razonante), el médico-psiquiatra francés Philippe Pinel publicó en 1801 un Tratado Médico-Filosófico sobre la Aliénation Mentale ${ }^{5}$ que, sin duda, dejó huella en los coetáneos y en los posteriores estudiosos de las enfermedades mentales. Pinel acuñó el término manie sans délire (manía/locura sin delirio) para referirse a los que hoy denominamos como psicópatas. Este autor subrayó que algunos pacientes realizaban actos impulsivos y auto-punitivos, aunque su capacidad de razonamiento estaba intacta y se daban cuenta de la irracionalidad de lo que estaban haciendo. Algunas de las palabras textuales que empleó Pinel en su libro de 1801 para caracterizar a los "locos sin delirio" eran las siguientes:

\footnotetext{
No me sorprendió en absoluto encontrar a muchos locos que no evidenciaban ninguna lesión de la capacidad de comprensión pero que estaban bajo el dominio de una furia abstracta e instintiva, como si únicamente las facultades del afecto hubiesen sufrido daño.
}

La manie sans délire fue, pues, el concepto más aproximado que empleó Pinel para caracterizar a los psicópatas de su época ${ }^{6}$. Con el término locura sin delirio, Pinel hablaba de personas que tienen todas las características de la manía pero que carecen del delirio. Él llamaba manía a los estados de furor persistentes y psicosis florida, muy distinto del concepto actual de manía que manejamos en 
Psicopatología. Asimismo, Pinel aclaraba lo siguiente con respecto al carácter específico de la manía sin delirio:

0 es continuo o se caracteriza por accesos o paroxismos periódicos. No se advierte ninguna alteración en las funciones del entendimiento, en la percepción, en el juicio, en la imaginación, en la memoria, pero sí cierta perversión en las funciones afectivas, un ciego impulso a cometer actos de violencia, o también un furor sanguinario, y esto sin que se pueda señalar ninguna idea dominante, ni ninguna ilusión de la imaginación que sea la causa determinante de estas funestas inclinaciones.

Desde antaño hasta Pinel se argumentó con fuerza la legitimidad de esta entidad psicopatológica, ya que era universalmente sabido que los trastornos mentales eran, lógicamente, trastornos de la mente. Como la mente era equivalente a la razón, sólo una desintegración de las facultades de la razón y del intelecto podía considerarse locura. No obstante, a partir de Pinel surgió la creencia de que se podía estar loco (manie) sin que existiese una confusión de la mente (sans délire). Así pues, parece claro que, aunque Pinel no empleó expresamente el término psicopatía, su ya clásico concepto de manía sin delirio fue una auténtica revolución conceptual adelantada a sus tiempos y que sentó parte de las bases teóricas de lo que actualmente entendemos por "psicópata". Las vívidas descripciones de este autor han tenido su seguimiento desde entonces hasta ahora, y con pocos matices ${ }^{6}$.

Treinta y cuatro años más tarde, en 1835, el médico-psiquiatra británico James Cowles Prichard publica su obra clave $A$ treatise on insanity and other disorders affecting the mind ("Un tratado sobre la insania y otros trastornos de la mente") ${ }^{7}$, en la que habla de la moral insanity (insania/locura moral) para referirse a estos sujetos (a los psicópatas). Aunque Prichard aceptaba la idea de Pinel de la locura sin delirio, disentía de la actitud moralmente neutra de Pinel hacia estos trastornos, y se convirtió en el principal exponente de la idea de que estos comportamientos significaban un defecto del carácter que había que reprender y merecía condena social.

Prichard también amplió la perspectiva del síndrome original, incluyendo bajo la calificación de locura moral una gran variedad de trastornos mentales y emocionales anteriores. Todos estos pacientes compartían un defecto común de la capacidad para guiarse a sí mismos según los "sentimientos naturales", es decir, un sentido intrínseco y espontáneo de rectitud, bondad y responsabilidad. Las personas con este trastorno estaban como poseídas, a pesar de que eran capaces de entender, desde el punto de vista intelectual, las diferentes elecciones posibles, pero otorgaban un mayor poder a los "afectos" que les impulsaban a llevar a cabo comportamientos socialmente reprobables. Las siguientes fueron las palabras textuales con las que Prichard describió a la moral insanity en 1835 :

Existe una forma de trastorno mental en el que las funciones intelectuales parecen estar intactas, mientras que el trastorno se manifiesta básica o únicamente por el estado de los sentimientos, temperamento o hábitos. En los casos de este tipo, los principios morales 0 activos de la mente están pervertidos o depravados; el poder de autodominio se ha perdido o está muy alterado, y el individuo es incapaz no sólo de hablar o razonar sobre los temas que se le proponen, sino de conducirse con decencia y propiedad en los asuntos de la vida.

La separación entre la locura debida a defectos del "razonamiento" y la debida a defectos de los "afectos naturales" expuesta por Prichard dio lugar a una controversia muy importante que se prolongó durante muchos años en los campos médico y legal. La entidad de Prichard de locura moral tenía poco en común como síndrome clínico con las ideas contemporáneas de psicopatía o personalidad antisocial. Un grupo tan diverso de trastornos fue subsumido en la categoría de Prichard, de forma que casi todas las alteraciones mentales, distintas del retraso mental y la esquizofrenia, se diagnosticarían así en la actualidad.

Tanto Pinel como Prichard luchaban contra la idea, imperante en esos tiempos, del filósofo John Locke, quien decía que no podía haber manía (furor, psicosis) sin delirio (es decir sin compromiso del intelecto). Por lo tanto, los jueces no declaraban insano a ninguna persona que no tuviera un compromiso intelectual manifiesto (delirio). Pinel y Prichard trataron de imponer el concepto de que existían insanias sin compromiso intelectual, pero sí afectivo y volitivo. Es decir, que las funciones mentales (intelecto, afectividad, voluntad) se podían enfermar de manera independiente.

Es evidente que Prichard no hablaba exactamente de lo que hoy consideraríamos una personalidad psicopática. Tampoco lo hicieron ni Pinel ni Benjamin Rush, este último un psiquiatra alemán que había propuesto en 1812 el término de inferioridad psicopática.

Pero, como decíamos antes, todos estos términos dejaron huella, una huella que afectó negativamente al concepto de psicopatía, ya que comenzó a llamárseles a estos sujetos con epítetos tan peyorativos y esperpénticos como los de imbéciles morales, idiotas morales, degenerados constitucionales, insanos morales, etc. En definitiva, al psicópata se le ha venido llamando de todo menos por su nombre. 
Sin lugar a dudas, este tipo de etiquetas y/o nomenclaturas terminológicas peyorativas fueron las responsables de irle creando al concepto de psicopatía una mala posición en las indagaciones de aquellos albores y una mala prensa en la actualidad. Es evidente que la psicopatía se ganó una muy mala reputación, precisamente, debido a la incapacidad de aquellos clínicos tanto por llegar a un consenso general -en cualquier caso, una empresa imposible en aquellos tiempos, con tantas escuelas o corrientes como había- como por la imposibilidad de delimitar científicamente las ostensibles hoy en día diferencias entre los psicópatas y los que lo parecen.

\section{Las personalidades psicopáticas: ¿o, más bien, psicopatologías de la personalidad?}

Como bien ha advertido el profesor García-Pablos de Molina en su Tratado de Criminología ${ }^{8}$, desde que en 1896, en la primera edición de su obra seminal, definiera Kraepelin la personalidad psicopática ${ }^{9}$, la doctrina psiquiátrica ha polarizado en torno a esta categoría buena parte del debate científico. En el momento de verificar posibles conexiones entre anomalía o trastornos psíquicos y crimen, el concepto de psicopatía ha ocupado un papel estelar, a pesar de que su delimitación no concite precisamente consenso alguno (con razón se ha dicho que no existe "el" psicópata ${ }^{10}$, ni dos psicópatas iguales): el número y la heterogeneidad de las personalidades psicopáticas (tipologías), la etiología muy diversa que se atribuye a tales cuadros clínicos y los rasgos de personalidad descritos en cada caso demuestran la complejidad y controversia del problema.

Fue el psiquiatra alemán Emil Kraepelin quien en 1903, con la séptima edición de su influyente obra Psychiatrie: Ein Lehrbuch ${ }^{11}$, vino a poner un poco de sentido al sinsentido histórico que el concepto de psicopatía había recorrido hasta entonces. Aunque seguían resonando ciertos ecos de la antaño manía/locura sin delirio, Kraepelin comenzó a hablar de los estados psicopáticos y, sobre todo, de las personalidades psicopáticas ${ }^{12,13}$. Ya en esta época, él fue quien primero advirtió que los psicópatas ni se encontraban recluidos dentro de los sanatorios mentales -los Ilamados, por aquel entonces, manicomios- ni tampoco sólo se circunscribían al submundo delincuencial.

Veinte años después, el doctor Kurt Schneider ${ }^{14}$, otro psiquiatra alemán y pupilo de Kraepelin, tomó el testigo de éste y lo plasmó en su ya clásica obra Die Psychopathischen Personlichkeiten ("Las Personalidades Psicopáticas"), sin duda la obra más influyente en los postulados teóricos y nosológicos de las posteriores clasificaciones psiquiátricas internacionales, tanto la de la American Psychiatric Association (APA) como la de la Organización Mundial de la Salud (OMS).

Lo que hizo Schneider fue poner de manifiesto que existían, para él, hasta diez subtipos de personalidades psicopáticas -hipertímicos, deprimidos, miedosos, fanáticos, vanidosos, lábiles, explosivos, fríos, abúlicos y asténicos-, subtipos que, curiosamente, se parecen sobremanera a los actuales diez trastornos de la personalidad recogidos en el DSM-IV-TR de la APA. Otra de las novedades que hizo notar este autor fue advertir sobre lo que ya había apuntado su mentor, es decir, que los psicópatas se encontraban también fuera de los contextos penitenciarios. En general, y muy conocida, la fórmula de Schneider ${ }^{14}$ definía las personalidades psicopáticas con una frase que ya ha quedado para la posteridad: "aquéllas que por su anormalidad sufren o hacen sufrir a la sociedad" (p. 17).

Aunque las personalidades psicopáticas de Kraepelin y de Schneider supusieron un cierto nuevo aire en la comprensión del concepto de psicopatía tal y como lo entendemos en la actualidad, quizás podríamos reprocharle a ambos el hecho de que enmarcaran la psicopatía dentro de un sistema psicopatológico en el que, en ningún caso, tiene hoy cabida este constructo, ya que, precisamente, una de las características esenciales de la psicopatía es la ausencia de manifestaciones psicopatológicas de cualquier tipo. Por tanto, Kraepelin y Schneider describieron, para ser más exactos, una serie de psicopatologías de la personalidad, más que personalidades psicopáticas sensu stricto.

\section{La sociología criminal y el psicoanálisis criminal}

\section{La sociología criminal o de la desviación: los sociópatas}

A finales de los años 20 y principios de los años 30 del pasado siglo, los sociólogos estudiosos de la conducta desviada y criminal no estaban en absoluto de acuerdo con el término "psicópata", de ahí que autores como George Everett Partridge propusiera el término sociopatía como sustituto para acabar con la polémica teórico-conceptual existente ${ }^{15,16}$.

En una revisión de la investigación y la teoría realizada durante los 50 años anteriores sobre la "categoría esquiva" de la personalidad psicopática, el 
conocido psiquiatra británico Sir Aubrey Lewis comentó lo siguiente ${ }^{17}$ :

Este trastorno revela una preocupación por el estado nosológico del concepto... sus implicaciones forenses, sus subdivisiones, sus límites [y] la propiedad de identificar la personalidad psicopática con un comportamiento antisocial. El efecto de la lectura de textos literarios aparentemente sólidos es descorazonador; existe una cantidad enorme de teorías inútiles y polémicas repetitivas, y una gran oscuridad terapéutica (pp. 137-138).

Setenta años antes ya se habían cuestionado los mismos aspectos, en especial si la personalidad psicopática era o no sinónimo del verdadero comportamiento antisocial. La revisión detallada de George Partridge ${ }^{16}$ sobre el concepto de la psicopatía, que prevalecería después, comienza del siguiente modo:

Las ideas relacionadas con la personalidad psicopática están ampliamente esparcidas en los ámbitos psiquiátrico y criminológico. Mucho de lo que se ha escrito es casi incidental cuando se estudia la delincuencia en general; algunos se refieren a los diferentes tipos de trastornos mentales en los que están implicadas las desviaciones de la personalidad (p. 53).

En relación con la cuestión de si la psicopatía y el comportamiento antisocial son una sola cosa, Partridge ${ }^{16}$ estableció lo siguiente:

Comparativamente, se ha prestado poca atención [psicopatológica] a las desviaciones de la personalidad que, aunque distintas, no se expresan en comportamientos antisociales.

Existe la creencia de que al menos algunos tipos crónicos de comportamientos desviados son las extensiones visibles de los aspectos profundos de la personalidad (p. 75).

Por otro lado, al comentar las relaciones halladas entre la psicopatía diagnosticada y las historias recogidas de comportamientos criminales o delincuentes, Partridge ${ }^{16}$ puntualizó así:

En la producción de la delincuencia en general, la importancia de la psicopatía ha recibido cierta atención. Hemos visto que algunos encuentran una gran proporción de personalidades psicopáticas en grupos criminales o entre los delincuentes en general, y algunos sólo parecen encontrar una pequeña proporción (p. 93).

En la conclusión de su análisis, Partridge se preguntó si la tendencia de los nosólogos (los psiquiatras de la época) a centrarse en los comportamientos antisociales, a expensas de la estructura profunda de la personalidad y sus variantes psicopáticas, reflejaba simplemente que estos comportamientos eran muy "obvios". A este respecto, Partridge ${ }^{16}$ sostenía lo siguiente:

\begin{abstract}
Una razón por la que se ha generado confusión sobre los llamados psicópatas es que, en estos casos, las desviaciones de la personalidad aparecen en edades muy tempranas y en una forma distinta... La principal diferencia... reside en que las formas sociopáticas son más objetivas simplemente en sus manifestaciones o patrones adaptativos, o al menos son las que más fácilmente pueden observarse (pp. 98-99).
\end{abstract}

\section{El psicoanálisis y sus explicaciones circulares: los delincuentes por sentimientos de culpa}

A medida que los nuevos conceptos y teorías del psicoanálisis se fueron consolidando durante la década de los años veinte, las nociones preliminares y más extendidas en relación con el carácter de los psicópatas fueron el objeto/tópico principal de las publicaciones de los clínicos, cada uno de los cuales partía de su escuela de pensamiento. La mayoría se vieron enfrascados en esta tarea gracias a un texto de Freud $^{18}$ titulado "Algunos tipos de carácter observados con el trabajo psicoanalítico". En este texto, Freud describió "actos peculiares" que no parecían ser propios del carácter del individuo. Al exponer la dinámica subyacente a un subgrupo de estos casos, refiriéndose a la "criminalidad a partir de un sentido de culpa" (los denominados delincuentes por sentimientos de culpa), Freud ${ }^{18}$ escribió lo siguiente:

El trabajo analítico lleva a la sorprendente conclusión de que tales acciones se producen precisamente porque están prohibidas y, al llevarlas a cabo, la persona disfruta de una sensación de alivio mental. Sufren una sensación aprensiva de culpa, de la que no conocen el origen, y, una vez que han cometido la acción prohibida, la opresión se mitiga (p. 342).

Este texto freudiano fue el desencadenante de numerosos trabajos clínicos de otros psicoanalistas y neopsicoanalistas. Entre los que se escribieron desde principios hasta mediados de los años veinte cabe citar el Joven Díscolo de Aichhorn, el estudio de Reich sobre El Carácter Esclavizado por el Impulso, el análisis de Abraham sobre la vida e Historia de un Impostor, y los trabajos de Alexander en torno al "carácter neurótico".

Aunque Partridge lo intentó y los psicoanalistas continuaban sin salir de sus clásicas explicaciones de tipo circular (como las califica Hare ${ }^{19}$ ), la propuesta de emplear el término sociopatía como sustituto del 
de psicopatía no llegó a buen puerto ${ }^{6}$. Si bien es cierto que actualmente los norteamericanos son asiduos empleadores del término sociopatía, el gremio científico, en general, sigue manteniendo la nomenclatura tradicional de psicopatía y han establecido sutiles diferencias. Así, mientras el problema subyacente del psicópata se encuentra en "algo" interno a su misma estructura de personalidad, el de los sociópatas descansaría, más bien, en la estructura de las características de la sociedad de cada momento.

Lejos de posicionarnos en uno u otro sentido, lo cierto es que ambos conceptos, si bien no son totalmente contradictorios, pueden emplearse -pero no indistintamente, ni tampoco como si fueran intercambiables- siempre y cuando estas diferencias se subrayen $^{20}$.

\section{La psicología criminal y forense: psicó- patas subclínicos versus psicópatas criminales}

Y esto es precisamente lo que vino (tal como se refleja en el subtítulo de su libro) a aclarar el doctor Hervey Milton Cleckley en 1941, con su ya clásica obra: The Mask of Sanity ${ }^{4}$. Tras la quinta edición de esta obra, en $1976^{21}$, fue el doctor Hare ${ }^{22-24}$ quien, posteriormente, elevara a la categoría de empíricas las observaciones y descripciones clínicas tan precisas que realizara Cleckley con el estudio de sus propios pacientes.

\section{Los psicópatas subclínicos o "socializados"}

Como decíamos, en 1941 apareció publicada la primera edición de esa obra, que, sin duda, marcaría un entonces y un después en la comprensión de la psicopatía como un constructo clínico-forense válido y fiable para los tiempos modernos y los nuevos estilos de ser y de vida. El doctor Cleckley puso el foco de atención en los aspectos personales y emocionales del psicópata, más que en sus características o rasgos conductuales, a los que otorgó su debida importancia, pero siempre en base a lo que ocurría en la mente y sentimientos de estos individuos.

Así, la nueva hipótesis o concepto que formuló Cleckley en 1941 fue el de afasia semántica. Este autor describía la mente y pensamiento del psicópata del siguiente modo ${ }^{4}$ :

Cuando examinamos a un psicópata, los procesos lógicos del pensamiento funcionan perfectamente (...). En un análisis de su discurso verbal, todos los juicios de valor y las apreciaciones emocionales son correctos (...). Sin embargo, esa capacidad aparente es incapaz de guiar de modo efectivo el comportamiento del sujeto: sólo cuando el sujeto conduce su vida podemos encontrar evidencia de lo poco que esa comprensión teórica que posee significa para él (...). Lo que tomamos como evidencia de su cordura no influirá de modo sustancial o consistente en su comportamiento.

El psicópata muestra la más absoluta indiferencia ante los valores personales, y es incapaz de comprender cualquier asunto relacionado con ellos. No es capaz de interesarse lo más mínimo por cuestiones que han sido abordadas por la literatura o el arte, tales como la tragedia, la alegría o el esfuerzo de la humanidad en progresar. También le tiene sin cuidado todo esto en la vida diaria. La belleza y la fealdad, excepto en un sentido muy superficial, la bondad, la maldad, el amor, el horror y el humor no tienen un sentido real, no constituyen una motivación para él. También es incapaz de apreciar qué es lo que motiva a otras personas. Es como si fuera ciego a los colores, a pesar de su aguda inteligencia, para estos aspectos de la existencia humana. Por otra parte, es inútil explicarle dichos aspectos, ya que no hay nada en su conocimiento que le permita cubrir esa laguna con el auxilio de la comparación. Puede, eso sí, repetir las palabras y decir que lo comprende, pero no hay ningún modo para que se percate de que realmente no lo comprende (pp. 90-91).

Ya lo expresaron más gráficamente Johns y Quay 25 cuando señalaron que el psicópata "se sabe la letra, pero no la música". Por tanto, llegados a este punto, se hace necesario distinguir en los psicópatas entre una conciencia intelectual y una conciencia moral' ${ }^{6}$, y siempre en referencia a las conductas que ejecutan. En pocas palabras, esta distinción se basaría en lo siguiente: saben lo que hacen, pero no les importan en absoluto las cicatrices psicológicas y emocionales que causan con sus actos. Entonces, ipodemos decir que falla algo dentro de la conciencia del psicópata? En caso de que fallare algo, ¿de qué se trataría? ¿Se tratará de un déficit cognitivo? ¿Se tratará de esa especie de demencia o afasia semántica? ¿Es posible que los psicópatas no piensen en voz alta? ¿Y en voz baja? Esta última es la verdadera conciencia, esa que todos conocemos y describimos generalmente como "una vocecilla interior que nos dice lo que está bien y lo que está mal", algo así como "el diablillo bueno y el malo", en suma: el centinela de nuestra conciencia.

Parece evidente que, en los psicópatas, la lógica del razonar sensatamente y de la conversación interior -en voz baja- permanecen ausentes. Incluso las conversaciones exteriores, dirigidas a los demás, de estos individuos encierran una gran multitud de 
incongruencias y de saltos inopinados de un tema a otro, como si parecieran, sin serlo, como los esquizofrénicos, los cuales presentan un descarrilamiento del lenguaje muy ostensible. Pero los psicópatas, insistimos, ni deliran, ni viven en otro mundo, ni han perdido el juicio.

Sin embargo, como acertadamente señala el profesor Vicente Garrido en su libro El Psicópata26, el problema en todo esto es que el psicópata puede enmascarar fácilmente esas disonancias 0 incongruencias lingüísticas mediante sus espectaculares dotes de manipulador y por su gran encanto superficial. Ahora, eso sí: en cierto modo podemos "detectar" algunas de esas incongruencias si seguimos y estamos atentos a un principio básico, esencial, con el que podemos desenmascararlo en buena medida: se trata, simple y llanamente, de comparar lo que dice con lo que hace.

Hay que tener en cuenta que los psicópatas presentan una enorme discrepancia entre su discurso verbal y sus actos. Esto es precisamente lo que llevó al doctor Cleckley a formular su conocida y difundida hipótesis de que el psicópata pudiera sufrir una especie de demencia o afasia semántica, postulando la existencia de "una anormalidad o defecto en un nivel profundo que perturba la integración y la apreciación normal de la experiencia". Tras formular su hipótesis, no tardaron los científicos de laboratorio en tratar de probar la verosimilitud de la misma a través de una pluralidad de técnicas y estudios de investigación biomédicos y psicológicos, pero siempre con muestras de psicópatas encarcelados (y, más en concreto, con delincuentes comunes, de ahí muchos de los resultados contradictorios en la investigación actual).

La hipótesis de Cleckley ${ }^{21}$ sobre la afasia semántica es muy sugerente, pero requeriría de muchos matices, objeto de análisis para un estudio aparte. En la presente revisión, lo que nos interesa son los 16 rasgos o características clínico-descriptivas que en la edición de 1976 nos ofreció este autor sobre los que él consideraba como psicópatas "subclínicos", descripciones que realizó tras largas observaciones de años de trabajo con pacientes de su propia consulta.

Huelga decir que, durante las últimas décadas, el psiquiatra norteamericano Hervey Cleckley se ha convertido en un referente principal para los estudiosos de la psicopatía ${ }^{27}$. Como decíamos, fue en la quinta edición de su The Mask of Sanity, publicada en 1976, donde el doctor Cleckley expuso y especificó estos 16 rasgos psicopáticos, haciendo la primera descripción comprehensiva del psicópata prototípico e intentando clarificar el problema de las terminologías y contrarrestar la tendencia a incluir bajo el rótulo de psicopatía trastornos muy diferentes. En la Tabla 1 sintetizamos el listado de dichas 16 características psicopáticas.

A diferencia de otras posturas anteriores, Cleckley ${ }^{21}$ consideraba que la psicopatía es un trastorno grave (más peligroso incluso que la psicosis) debido a su apariencia externa de normalidad. Para este autor, el comportamiento antisocial de los psicópatas es sólo una de las posibles manifestaciones de un síndrome clínico más amplio, cuyo núcleo está representado por una serie de características distintivas a
1. Encanto superficial y notable inteligencia.

2. Ausencia de delirios y de otros signos de pensamiento irracional.

3. Ausencia de nerviosismo y de manifestaciones psiconeuróticas.

4. Poco fiable, indigno de confianza, irresponsable.

5. Falsedad o insinceridad.

6. Incapacidad para experimentar remordimiento o vergüenza.

7. Conducta antisocial sin motivo aparente o que la justifique.

8. Falta de juicio y dificultades para aprender de la experiencia.
9. Egocentrismo patológico e incapacidad de amar.

10. Pobreza o escasez generalizada de reacciones afectivas básicas.

11. Pérdida específica de insight (intuición).

12. Insensibilidad en las relaciones interpersonales ordinarias.

13. Conducta desagradable y exagerada bajo los efectos del alcohol y, a veces, sin él.

14. Amenazas de suicidio raramente consumadas.

15. Vida sexual impersonal, frívola y poco integrada o estable.

16. Incapacidad para seguir cualquier plan de vida.
Tabla 1.

Los 16 rasgos o características clínicas del psicópata, según Hervey M. Cleckley (1976). 
nivel emocional e interpersonal. Es por ello que este autor concibió al psicópata como un individuo de trato social aparentemente agradable pero altamente asocial, superficial e impulsivo.

Aunque algunos de los criterios, ítems o rasgos psicopáticos formulados por Cleckley son indicadores de desviación social, este autor mantenía que la conducta antisocial y destructiva por sí misma no era suficiente para establecer un diagnóstico de psicopatía, aconsejando distinguirla de la criminalidad común. De hecho, contempló la posibilidad de que las personalidades psicopáticas no se encuentren únicamente en las instituciones penitenciarias, sino también en algunas de las posiciones sociales más respetadas, como científicos, médicos, psiquiatras, juristas y hombres de negocio ${ }^{21}$.

Cleckley se refirió a estos casos como manifestaciones subclínicas del trastorno al considerar que el concepto de "trastorno" implicaba un cierto grado de incapacidad o desajuste social. Sin embargo, enfatizó que se trataba de manifestaciones alternativas de la misma patología que subyace a los casos clínicos, ya que los casos subclínicos muestran signos de experimentar reacciones internas similares a las de los casos clínicos. Es por esta razón por la que, en términos alegóricos, sugirió que el psicópata padece demencia semántica, pues no es capaz de comprender las experiencias genuinamente humanas en profundidad, aunque finge entenderlas. Es decir, el psicópata es capaz de imitar la moral y otros sentimientos sutiles del ser humano y tampoco tiene dificultad alguna en entender las normas de la interacción social, pero le faltan las emociones asociadas a dichas reglas.

La existencia de los psicópatas "subclínicos" que Cleckley puso de relieve, como veremos luego, dio lugar a una sucesiva e imparable retahíla de términos que vienen a ser la misma entidad. Según este autor, lo que verdaderamente diferencia a los psicópatas que continuamente entran y salen de las prisiones o los hospitales psiquiátricos y a los psicópatas "con éxito" es que, en estos últimos, la apariencia de normalidad es más firme ${ }^{21}$.

\section{Los psicópatas criminales o "puros"}

El legado de los 16 criterios de la psicopatía propuestos por Cleckley es innegable. Estos rasgos han sido utilizados como un tipo de definición operativa de la psicopatía, en un principio, para realizar evaluaciones clínicas globales y, posteriormente, como un listado o conjunto de escalas de evaluación ${ }^{28,29}$.
Fue precisamente el doctor Robert D. Hare, profesor emérito en la Facultad de Psicología de la Universidad de British Columbia (Canadá), quien, partiendo de las características señaladas por Cleckley, fue elaborando su conocido instrumento de evaluación de la psicopatía desde 1980 (Ilamado PCL ${ }^{28,29,30,31}$ ) hasta 1991 que fue publicado formalmente con el nombre de Psychopathy Checklist-Revised (PCL-R ${ }^{23}$ ), del que recientemente, en 2003, se ha publicación su segunda edición incluyendo datos de población reclusa femenina ${ }^{24}$.

En términos operativos, el concepto de psicopatía aportado por Hare en su PCL-R se distingue de otros trastornos o cuadros psicopatológicos por un patrón característico de síntomas en tres planos o niveles: afectivo, interpersonal y conductual 1,19,32. Así, en el plano afectivo, estos individuos se caracterizan por experimentar emociones lábiles y superficiales, por su falta de empatía, de ansiedad y de sentimientos genuinos de culpa o remordimiento, así como por su incapacidad para establecer vínculos duraderos con personas, principios u objetivos. En el plano interpersonal, son arrogantes, egocéntricos, manipuladores, dominantes y enérgicos. Finalmente, en el plano conductual, son irresponsables, impulsivos y buscadores de sensaciones; suelen trasgredir con facilidad las normas sociales, y se caracterizan por un estilo de vida socialmente inestable que incluye comportamientos parasitarios y faltos de planificación. Entre las expresiones más obvias de estas tendencias de personalidad, Hare y su grupo destacaron la conducta criminal, el abuso de sustancias y el fracaso en cumplir con las obligaciones sociales o en hacerse cargo de las responsabilidades ${ }^{28,33}$.

En la Tabla 2 exponemos los primeros 22 ítems originales de la psicopatía que sirvieron a Hare para que, entre 1980 y 1985, elaborase un borrador del instrumento de evaluación de la psicopatía (Ilamado $\mathrm{PCL}$ ) con el cual se hicieron numerosas investigaciones que, posteriormente, dieron lugar a su revisión (el PCL-R).

Como decíamos, tras este primer listado original de 22 ítems psicopáticos, Hare y su grupo fueron realizando varios cambios en el listado para construir uno que fuera sencillo de utilizar. Estos cambios incluyeron eliminar dos ítems, uno porque era difícil de puntuar (ítem 22, "Abuso de drogas o alcohol no causado directamente por la conducta antisocial") y el otro porque proporcionaba poca información útil (ítem 2, "Diagnóstico previo de psicopatía"). El ítem 16 ("Conducta irresponsable como padre") fue cambiado por "Irresponsabilidad" en general. Otros títulos también fueron levemente modificados sin alterar la naturaleza de la característica de conducta a la 
que se refiere el ítem. Los ítems y procedimientos de puntuación actuales están descritos con más detalle que anteriormente, y algunas dificultades y aparentes inconsistencias en los criterios de puntuación fueron convenientemente revisadas. El resultado de todas estas revisiones fue el actual Listado de Psicopatía de 20-ítems.

Teniendo en cuenta todo este perfil en sus tres planos y el original PCL de los años ochenta, el doctor Hare comenzó a elaborar su actual PCL-R, en el cual se encuentran explicitados los vigentes 20 rasgos esenciales del psicópata criminal o "puro". Dado que la psicopatía enmarcada en el PCL-R puede entenderse también como una dimensión o como un continuo -en el que los sujetos van desde nada psicopáticos (0 puntos) hasta psicópatas puros (40 puntos)-, las variaciones de "psicópatas" que pueden surgir son múltiples, muy variadas y, en absoluto, nada desdeñables. En cualquier caso, el concepto completo de psicopatía es, justamente, ése que viene caracterizado por los citados 20 rasgos que al principio, en $1991^{23}$, fueron divididos en dos factores y que más modernamente, en $2003^{24}$, ha seguido la misma estructura factorial, pero con la novedad de haberlos separado por facetas y de haber incluido datos sobre población penitenciaria femenina.

En la Tabla 3 se expone el listado exacto de los 20 rasgos psicopáticos según las facetas a que corresponden.
1. Locuacidad / Encanto superficial.

2. Diagnóstico previo de psicopatía (o similar).

3. Egocentrismo / Sensación grandiosa de la autovalía.

4. Propensión al aburrimiento / Baja tolerancia a la frustración.

5. Mentira patológica y decepción.

6. Dirección / Falta de sinceridad.

7. Falta de remordimiento y culpabilidad

8. Falta de afecto y escasa profundidad emocional.

9. Insensibilidad / Falta de empatía.

10. Estilo de vida parásito.

11. Colérico / Falta de control conductual.
12. Relaciones sexuales promiscuas.

13. Problemas de conducta precoces.

14. Falta de metas realistas a largo plazo.

15. Impulsividad.

16. Conducta irresponsable como padre o madre.

17. Relaciones maritales frecuentes.

18. Delincuencia juvenil.

19. Elevado riesgo bajo parole o probation.

20. Incapacidad para aceptar la responsabilidad de sus actos.

21. Varios tipos de delitos.

22. Abuso de drogas o alcohol no directamente causados por la conducta antisocial.
Tabla 2.

Los 22 rasgos o características forenses del psicópata, según Robert D. Hare (1980, 1985).

Tabla 3.

Los 20 rasgos o características del psicópata "puro", según Robert D. Hare (2003). 
La suma de las evaluaciones individuales de los ítems proporciona una puntuación total en el PCL-R entre 0 y 40, indicativa del número de rasgos y conductas psicopáticas mostradas por un determinado sujeto ${ }^{34}$, y representativa del grado en que éste se aproxima al psicópata prototípico descrito por Cleckley $^{4,21}$. Según el autor de este instrumento de evaluación, una puntuación igual o superior a 30 es la puntuación de corte a partir de la cual se puede establecer el diagnóstico de psicopatía; asimismo, las puntuaciones medias (iguales o inferiores a 29) podrían estar indicando graves trastornos psicopatológicos y/o psiquiátricos (por ejemplo, esquizofrenia paranoide) $)^{23,32}$

Uno de los aspectos más importantes de este listado de 20 rasgos psicopáticos es que el concepto de psicopatía está operacionalizado empíricamente a través del PCL-R, siendo el único instrumento de evaluación de la psicopatía fiable y valido en contextos penitenciarios, que fue para la población para la que se creó; existen otros instrumentos derivados de éste e igualmente válidos y fiables para ser utilizados en contextos forenses y civiles con población adulta (PCL:SV ${ }^{35}$; P-SCAN ${ }^{36}$; B-SCAN ${ }^{37,38,39}$ ), así como con población infanto-adolescente (CPS ${ }^{40}$ y APSD $^{41}$ ) y juvenil (PCL:YV42).

Cualquier otra escala que dice pretender medir psicopatía no lo es tal (como, por ejemplo, la ya clásica escala Pd o de desviación psicopática del MMPI), de modo que establecer un juicio clínico-forense de "psicopatía" a través del empleo de este tipo de escalas ni es correcto ni es psicopatía sensu stricto; a lo sumo podría ser un indicativo de tendencias psicopáticas, las cuales habría que contrastarlas y demostrarlas a través del uso de los PCL.

\section{Regreso al presente y futuro: una reformulacion necesaria}

\section{Las diversas tipologías o subtipos de psicópatas}

Ante todo este panorama, es posible que las numerosas tipologías existentes de psicópatas tampoco hayan contribuido a clarificar el concepto más general de psicópata, el cual sigue siendo actualmente impreciso para algunos autores. Lamentablemente, los amplios esfuerzos doctrinales de, por ejemplo, Michael J. Craft han sido desoídos con frecuencia. En su influyente obra de 1966 Psychopathic Disorders and Their Assessment, Craft ${ }^{43}$, después de revisar el desarrollo histórico de esta categoría tan ambigua, concluyó que dos son los rasgos distintivos de una personalidad psicopática: 1) una incapacidad para responder emocionalmente en situaciones en las que se esperaría alguna respuesta, tratándose de una personalidad normal; y 2) una irresistible tendencia a actuar impulsivamente. En base a esto, el autor estableció que de estos dos rasgos distintivos generales se derivarían otros rasgos secundarios: agresividad, ausencia de sentimiento de culpa, falta de motivación o pulsión positiva, no influenciabilidad por el temor al castigo, etc.

Sin embargo, recientemente se han venido realizando diversos estudios empíricos que han probado que las tipologías de psicópatas no sólo son diversas sino, además, necesarias. Tal es el caso de, por ejemplo, la investigación llevada a cabo por Hugues F. Hervé, J. Yong Hui Ling y Robert D. Hare, presentada como ponencia en una Conferencia celebrada en New Orleans bajo el título de La Psicopatía Criminal y Subtipos ${ }^{44}$.

Partiendo de la base de que el concepto de psicopatía contenido en el PCL-R se compone de las cuatro facetas anteriormente expuestas, se podría hablar de subtipos de psicópatas. Al mismo tiempo, considerando que este instrumento consta de 20 ítems o rasgos, existen distintas combinaciones que pueden llevar a que se obtenga un puntaje elevado (igual o mayor que 30), debiendo tener al menos 2 puntos en 10 ítems y 1 en los demás. Como ya estableciera Hare $^{1}$, la psicopatía puede considerarse, en vistas de la polémica generada al respecto, tanto una categoría discreta (o taxón) como una categoría continua (es decir, un continuo dimensional que, según las puntuaciones del PCL-R puede ir desde más hasta menos psicopático). En consecuencia, no todas las personas que delinquen y que obtienen un puntaje elevado en el PCL-R son iguales.

Para demostrar esta hipótesis, Hervé, Ling y Hare ${ }^{44}$ llevaron a cabo un análisis de los resultados obtenidos en reclusos con puntajes elevados en el PCL-R. De acuerdo al puntaje obtenido por ellos en cada una de las cuatro facetas, identificaron tres grupos o subtipos de psicopatía y un cuarto grupo de pseudopsicópatas o sociópatas. Este estudio tuvo sus consecuencias posteriores, ya que le valió a Hare para que puliera su PCL-R y publicara en 2003 su segunda edición. Los 3 subtipos de psicópatas hallados fueron los siguientes:

- Clásico o prototípico: puntaje elevado en las cuatro facetas.

- Manipulador: puntaje alto en la Faceta 1 (Interpersonal) y en la Faceta 2 (Afectiva), y más bajo en las otras dos facetas.

- "Macho": puntaje bajo en la Faceta 1 y alto en las demás. 
Tras los resultados obtenidos del total de la muestra, un $32 \%$ correspondía al psicópata clásico o prototípico, un 25\% al manipulador, un $27 \%$ al "macho" y un $16 \%$ al pseudopsicópata o sociópata. El hallazgo más destacado fue que los tres primeros subtipos habían obtenido un alto puntaje en la Faceta 2 (Emocional, Afectiva), mientras que los pseudopsicópatas o sociópatas eran completamente distintos, habiendo obtenido un puntaje significativamente inferior en esta faceta.

Una de las principales implicaciones de este estudio se relaciona con la posibilidad de tratamiento de estos sujetos. Dado que la psicoterapia apela principalmente a las emociones, ésta podría resultar más efectiva para el grupo de los sociópatas que para los tres subgrupos restantes, debido a sus características emocionales $^{45}$. En este sentido, resulta evidente que el puntaje obtenido en el PCL-R es relevante no sólo para evaluar la respuesta a los tratamientos psicoterapéuticos, sino también, como pasamos a ver a continuación, como factor con gran capacidad predictiva del riesgo de peligrosidad, violencia y reincidencia ${ }^{46}$.

\section{Los modernos psicópatas "predelincuentes", "exitosos" o "integrados"}

Decíamos anteriormente que los psicópatas "subclínicos" observados por Cleckley en su propia consulta generaron un gran interés (que aún hoy permanece vigente, incluso con mayor ímpetu) en conocer si existen los que más modernamente han sido denominados como psicópatas "predelincuentes" o "subcriminales"19, psicópatas "exitosos" o "con éxito"3739,47-51 o psicópatas "integrados" 6,20,52, es decir, individuos psicopáticos (con varios rasgos de la psicopatía) que, sin ser delincuentes, logran triunfar en profesiones socialmente bien consideradas $2,53,54$.

En su influyente libro Without Conscience, el doctor Hare ${ }^{19}$ vino a poner en conocimiento de la sociedad en general la existencia de este tipo de individuos, a los que él califica de "predelincuentes". Estableciendo la importantísima diferencia con los delincuentes comunes multireincidentes, el propio autor, en extensión, asevera lo siguiente:

Muchos psicópatas se pasan la vida entrando y saliendo de cárceles y correccionales. La pauta característica es ir de un trabajo u otro a la prisión y después vuelta a las calles, vuelta a la prisión (quizás a un centro de salud mental) y después fuera otra vez. En los hospitales psiquiátricos no suelen pasar mucho tiempo ya que, muy pronto, el personal se da cuenta de que el psicópata sólo da problemas e interrumpe la rutina institucional. Son como pelotas de ping pong fuera de control.

Sin embargo, muchos psicópatas nunca ingresan en la cárcel ni en ningún otro centro. Parece que funcionan razonablemente bien -son abogados, médicos, psiquiatras, mercenarios, oficiales de policía, líderes religiosos, militares, hombres de negocios, escritores, artistas y demás-, sin contravenir la ley o, al menos, sin que les cojan. En realidad, son tan egocéntricos, insensibles y manipuladores como el resto de psicópatas; sin embargo, su inteligencia, su familia, sus habilidades sociales y sus circunstancias les permiten construir una fachada de normalidad y obtener lo que desean con relativa impunidad.

Algunos estudiosos los Ilaman "psicópatas con éxito". Otros opinan que individuos de esa clase benefician a la sociedad. Según este argumento, debido a que son capaces de ignorar las normas sociales, los psicópatas inteligentes pueden trascender los límites del pensamiento convencional, aportando una chispa de creatividad a las artes, el teatro, el diseño y demás. Bajo mi punto de vista, por mucho que aporten, lo importante son los corazones rotos que dejan, las carreras que destrozan y la gente utilizada que dejan en el camino, $y$ todo en nombre de su necesidad de "expresar su verdadero yo".

Antes que llamarlos psicópatas con éxito -después de todo, su éxito es frecuentemente ilusorio y siempre a expensas de otros-, prefiero darles el apelativo de psicópatas predelincuentes [subcriminales]. Su conducta, aunque técnicamente no ilegal, violan los criterios éticos que tenemos la mayoría y se coloca en esa zona gris de la ley. Los psicópatas predelincuentes exhiben la misma conducta y actitud en todas las áreas de su vida, a diferencia de la gente que conscientemente adopta una actitud egoísta y sin escrúpulos en sus negocios, pero que son razonablemente honestos en otras áreas de su vida. Si mienten y engañan en su trabajo -y salen bien parados de ello e incluso los admiran- mentirán y engañarán en otras áreas de su vida (pp. 113-114).

Como decimos, a estos mismos sujetos, otros autores los han denominado psicópatas "con éxito" o psicópatas "integrados". Así, independientemente del calificativo que emplees para denominarlos, este tipo de sujetos tienen siempre el mismo perfil de personalidad: todos aquellos psicópatas adultos que hasta el momento de su detención o hasta que los desenmascararon pasaban por ciudadanos ejemplares, en realidad, no aparecieron ni aparecen de la nada: "eran las mismas personas antes y después de su detención y/o desenmascaramiento. Son psicópatas ahora y eran psicópatas antes"19. 


\section{Conclusiones y discusión}

La reformulación del concepto de psicopatía por la Psicología Criminal y Forense y su separación respecto de la tradición médico-psiquiátrica ha tenido lugar a lo largo de un proceso histórico cuyos primeros albores apuntan exactamente en el momento en que Cleckley comienza a estudiar a los psicópatas que le llegan a su clínica privada, cobrando la psicopatía una verdadera realidad psicosocial y entidad propia en el mismo momento en que el doctor Hare retoma los estudios de aquél y los separa del enfoque psiquiátrico y psicopatológico en el que había estado encapsulado desde antaño. De esta forma, la psicopatía pasa de ser un concepto nosológico excesivamente amplio, vago y difuso, aceptado y rechazado simultáneamente por la APA, a ser un constructo psicológico válido y fiable para los nuevos tiempos. Así, con la aparición del PCL-R, el constructo de psicopatía queda definitivamente operacionalizado, cobra un auténtico valor empírico que antes era meramente descriptivo sólo a nivel clínico.

Sin duda alguna, como hemos visto, el concepto de psicopatía ha sido una fuente constante de controversia y confusión ${ }^{55}$ a lo largo de los distintos períodos históricos ${ }^{56}$. Sin embargo, las implicaciones psicosociales, criminológicas y legales de esta anomalía de la personalidad no son nada desdeñables y debemos contribuir a la investigación para que las causas de la misma sigan cobrando aún mayor claridad científica ${ }^{57-59}$.

En vistas de la actual discordancia mantenida sobre el constructo de psicopatía por nuestra Jurisprudencia y Doctrina (tema que abordaremos en una Parte II de esta amplia revisión y análisis), quizá no seamos muy atrevidos al aseverar que buena parte de la "culpa" de esta situación descansa, paradójicamente, en el también disenso, aunque en mucha menor medida, que aún sigue existiendo entre ciertos sectores clínicos y el gremio de investigadores que dedican sus esfuerzos al estudio de la psicopatía. De ser así, el problema debiera solucionarse de inmediato consensuando posturas aisladas y minoritarias muy alejadas del consenso generalizado.

Tampoco podemos anclarnos en la errónea premisa que suele hacerse muchas veces de que todos los estudios de investigación sobre la psicopatía están realizados con muestras fundamentalmente norteamericanas, puesto que el PCL-R está publicado en inglés y baremado para la población citada. Esto no es cierto: Ios estudios realizados en España con psicópatas, si bien no son tan numerosos como los foráneos, existen ${ }^{53,59-63}$, por no mencionar el hecho de que el PCL-R ha sido convenientemente estandarizado para muestras penitenciarias españolas hace ya 10 años $^{64}$, estando actualmente publicado por TEA Ediciones en España.

Hoy por hoy, la división de la psicopatía en una vertiente emocional-afectiva anómala y otra vertiente conductual antisocial constituye un logro irrenunciable de los diversos estudios de Hare et al, así como también de todos aquellos estudios en los que se ha empleado convenientemente el PCL-R como procedimiento único de evaluación y diagnóstico de la psicopatía. Habiéndose demostrado en reiteradas ocasiones que psicopatía y TAP no son la misma entidad $^{65-70}$, no es aventurado decir que la psicopatía absorbe al TAP, es decir, que el TAP queda comprendido dentro de los rasgos o ítems del Factor 2 del PCL-R, aunque no necesariamente lo asume como imprescindible para la calificación o diagnóstico de psicopatía. En este sentido, quizás no insistamos lo suficiente cuando volvemos a recordar que psicopatía no es sinónimo de criminalidad o, en otras palabras, que ni todos los psicópatas son delincuentes, ni todos los delincuentes son psicópatas ${ }^{63}$.

\section{Bibliografía}

1. Hare RD. Psychopathy: A clinical construct whose time has come. Crim Justice Behav. 1996;23:25-54.

2. Torrubia R, Cuquerella A. Psicopatía: una entidad clínica controvertida pero necesaria en psiquiatría forense. Rev Esp Med Legal. 2008;34:25-35.

3. Moltó J, Poy R. La psicopatía: un constructo necesario en la Psicología Jurídica. En: Clemente M, Núñez J, editores. Psicología jurídica penitenciaria
(Vol. II). Madrid: Fundación Universidad-Empresa; 1997. p. 291-317.

4. Cleckley HM. The mask of sanity: an attempt to clarify the so-called psychopathic personality. $1^{\text {a }}$ ed. St. Louis: Mosby; 1941.

5. Pinel P. Traité médico-philosophique sur l'aliénation mentale ou la manie. Paris: J. A. Brosson; 1801.

6. Pozueco Romero JM. Psicópatas integrados: Perfil psicológico y personalidad. Madrid: EOS (Colección de Psicología Jurídica); 2010. 
7. Prichard JC. A treatise on insanity and other disorders affecting the mind. London, UK: Sherwood, Gilpert \& Piper; 1835.

8. García-Pablos de Molina A. Tratado de criminología. $3^{a}$ ed. Valencia: Tirant lo Blanch; 2003.

9. Kraepelin E. Psychiatrie: Ein Lehrbuch. 5a ed. Leipzig: Barth; 1896.

10. Göppinger H. Criminología. Madrid: Reus; 1975.

11. Kraepelin E. Psychiatrie: Ein Lehrbuch. $7^{\mathrm{a}}$ ed. Leipzig: Barth; 1903.

12. Dolan B, Coid J. Psychopathic and antisocial personality disorders. London: Gaskell; 1993.

13. Meloy JR. The psychopathic mind: origins, dynamics, and treatment. New Jersey: Jason Aronson; 1988.

14. Schneider K. Die psychopathischen persönlichkeiten. Viena: Deuticke; 1923.

15. Partridge GE. A study of 50 cases of psychopathic personality. Am J Psychiatry. 1927;7:953-74.

16. Partridge GE. Current conceptions of psychopathic personality. Am J Psychiatry. 1930;10:53-99.

17. Lewis A. Psychopathic personality: A most elusive cathegory. Psychol Med. 1974;4:133-40.

18. Freud S. Some character-types met with in psychoanalitic work (Traducción de E.C. Mayne). En: Collected Papers (Vol. 4). London: Hogart Press (Trabajo original publicado en 1916); 1925; p. 318-344.

19. Hare RD. Without conscience: The disturbing world of the psychopaths among us. New York: Pocket Books; 1993.

20. Pozueco Romero JM. Psicopatía, trastorno mental y crimen violento: Aspectos clínico-forenses, médicolegales y criminológicos. Madrid: EOS (Colección de Psicología Jurídica); 2011.

21. Cleckley HM. The mask of sanity: an attempt to clarify the so-called psychopathic personality. $5^{\mathrm{a}}$ ed. St. Louis: Mosby; 1976.

22. Hare RD. A research scale for the assessment of psychopathy in criminal populations. Person Individ Dif. 1980;1:111-9.

23. Hare RD. The Hare Psychopathy Checklist-Revised. Toronto: Multi-Health Systems; 1991.

24. Hare RD. The Hare Psychopathy Checklist-Revised. $2^{a}$ ed. Toronto: Multi-Health Systems; 2003.

25. Johns JH, Quay HC. The effect of social reward on verbal conditioning in psychopathic and neurotic military offenders. J Consult Psychol. 1962;36:217-20.

26. Garrido VJ. El psicópata: Un camaleón en la sociedad actual. Alcira: Algar; 2000.

27. Patrick CJ. Back to the future: Cleckley as a guide to the next generation of psychopathy research. En:
Patrick CJ, editor. Handbook of psychopathy. New York: Guilford Press; 2006. p. 605-17.

28. Hare RD. A research scale for the assessment of psychopathy in criminal populations. Person Individ Dif. 1980;1:111-9.

29. Hare R.D. Comparison of procedures for the assessment of psychopathy. J Consult Clin Psychol. 1985;53:7-16.

30. Hare RD. Checklist for the assessment of psychopathy in criminal populations. En: Ben-Aron MH, Hucker SJ, Webster CD, editores. Clinical Criminology. University of Toronto, ON: Clarke Institute of Psychiatry; 1985. p. 157-67.

31. Hare RD. The Psychopathy Checklist. Vancouver: Department of Psychology, University of British Columbia. (Manuscrito no publicado). Vancouver, BC: Canadá; 1985.

32. Hart SD, Hare RD, Harpur TJ. The Psychopathy Checklist-Revised (PCL-R): An overview for researchers and clinicians. En: Rosen JC, McReynolds P, editores. Advances in psychological assessment (Vol. 8). New York: Plenum Press; 1992. p. 103-30.

33. Hare RD. Psychopathy and crime. En: L. Otten, editor. Colloquium on the correlates of crime and the determinants of criminal behavior. McClean, VA: The Mitre Corporation; 1980; p. 95-132.

34. Hare RD, Hart SD. Psychopathy, mental disorder, and crime. En: Hodgins S, editor. Mental disorder and crime. Newbury Park: Sage; 1993; p. 104-15.

35. Hart SD, Cox DN, Hare R D. Manual for the Screening Version of the Hare Psychopathy Checklist-Revised (PCL:SV). Toronto: Multi-Health Systems; 1995.

36. Hare RD, Hervé HF. The Hare P-Scan: Research Version. Toronto: Multi-Health Systems; 1999.

37. Babiak P. Psychopaths in the organization: Some thoughts for the HR professional. Ponenia presentada en la Society for Human Resource Management. Danbury, CT: Western Connecticut Chapter; 1998.

38. Babiak P. Psychopathic manipulation at work. En: Gacono CB, editor. The clinical and forensic Assessment of Psychopathy: a practitioner's guide. New Jersey: Lawrence Erlbaum Associates; 2000; p. 287-311.

39 Babiak P, Hare RD. Snakes in suits: When psychopaths go to work. New York: Regan Books; 2006.

40. Lynam DR. Pursuing the psychopath: Capturing the psychopath in a nomological net. J Abnorm Psychol. 1997; 106:425-38.

41. Frick PJ, Hare RD. Antisocial Process Screening Device (APSD). Toronto: Multi-Health Systems; 2002.

42. Forth AE, Kosson DS, Hare RD. The Hare Psychopathy Checklist: Youth Version (PCL:YV). Toronto: MultiHealth Systems; 1996/2003. 
43. Craft MJ, editor. Psychopathic disorders and their assessment. Oxford: Pergamon Press; 1966.

44. Hervé HF, Ling JYH, Hare RD. Criminal psychopathy and subtypes. Ponencia presentada en la Conferencia Bianual de la American Psychology-Law Society. New Orleans, LA, March 9-12, 2000.

45. Hare RD. Psychopathy: Its nature and implications for mental health and criminal justice. Ponencia presentada en el seminario Concepto de Psicopatía, sus Características, Evaluación e Implicancias en Servicios de Salud Mental y Judicial. Seminario organizado por la Gendarmería de Chile, Ministerio de Justicia, Gobierno de Chile, Asociación Chilena de Psicoterapias Cognitivas y Asociación Chilena de Psicología Jurídica. Santiago de Chile, Chile; 2004.

46. Hare RD. Psychopathy and risk for recidivism and violence. En: Gray N, Laing J, Noaks L, editores. Criminal justice, mental health, and the politics of risk. London: Cavendish Publishing; 2002; p. 27-47.

47. Babiak P. When psychopaths go to work: A case study of an industrial psychopath. Appl Psychol. 1995;44:171-88.

48. Babiak P. Psychopathic manipulation in organizations: Paws, patrons, and patsies. Ponencia presentada en el NATO Advanced Study Institute on Psychopathy; 1995.

49. Babiak P. Psychopathic manipulation in organizations: Pawns, patrons, and patsies. En: Cooke DJ, Forth AE, Newman JP, Hare RD, editores. Issues in criminological and legal psychology: No. 24, International perspectives on psychopathy. Leicester: British Psychological Society; 1996; p. 12-7.

50. Babiak P. Psychopathic personalities in organizations. Ponencia presentada en la Metropolitan New York Association for Applied Psychology. New York; 1996.

51. Hare RD, Babiak, P. Snakes in suit: When psychopaths go to work. Ponencia presentada en el Curso sobre Avances Actuales de la Psicopatía. Barcelona: Centre d'Estudis Jurídics i Formació Especialitzada, noviembre de 2004; 2004.

52. Garrido VJ. Cara a cara con el psicópata. Barcelona: Ariel; 2004.

53. Tormo Map. Validez del modelo de las cuatro facetas de la psicopatía de R.D. Hare (2003) en una muestra penitenciaria: Evidencia desde el laboratorio psicofisiológico. Castellón, España: Tesis Doctoral, Facultad de Ciencias Humanas y Sociales, Departamento de Psicología Básica, Clínica y Psicobiología, Universitat Jaume I; 2007.

54. Widiger TA, Corbitt EM. Antisocial Personality disorder: Proposals for DSM-IV. J Pers Disord. 1993;7:63-77.

55. Hare RD. Psychopathy and antisocial personality disorder: A case of diagnostic confusion. Psychiatr Times. 1996;13:39-40.
56. Cooke DJ, Forth AE, Hare RD, editores. Psychopathy: Theory, research and implications for society. The Netherlands: Kluwer Academic Publishers; 1998.

57. Cooke DJ, Forth AE, Newman JP, Hare RD, editores. Issues in criminological and legal psychology: No. 24, International perspectives on psychopathy. Leicester, UK: British Psychological Society; 1998.

58. Gacono CB, editor. The clinical and forensic assessment of psychopathy: a practitioner's guide. New Jersey: Lawrence Erlbaum Associates; 2000.

59. Moltó J, Poy R, Segarra P, Pastor MacC, Montañés S. Response perseveration in psychopaths: Interpersonal/affective or social deviant traits? J Abnorm Psychol. 2007;116:632-37.

60. Moltó J, Poy R, Pastor MacC, Montañés S, Segarra P, Tormo MaP. Emoción y psicopatía: Estudio experimental sobre el déficit en el procesamiento de información emocional con internos del Centro Penitenciario de Castellón. Castellón: Fundación Dávalos-Fletcher; 2001.

61. Pastor MC. Modulación del reflejo de sobresalto y medidas autonómicas en psicópatas encarcelados. Castellón: Tesis Doctoral, Facultad de Ciencias Humanas y Sociales, Departamento de Psicología Básica, Clínica y Psicobiología, Universitat Jaume I; 2001.

62. Pastor $M^{\mathrm{a}} \mathrm{C}$, Moltó J, Vila J, Lang PJ. Startle reflex modulation, affective ratings and autonomic reactivity in incarcerated Spanish psychopaths. Psychophysiology. 2003;40:934-38.

63. Raine A, Sanmartín J, editores. Violencia y psicopatía. Barcelona: Ariel; 2000.

64. Moltó J, Poy R, Torrubia R. Standarization of the Hare Psychopathy Checklist-Revised in a Spanish prison simple. J Pers Disord. 2000;14:84-96.

65. Williamson SE, Harpur TJ, Hare RD. Abnormal processing of affective words by psychopaths. Psychophysiology. 1991;28:260-73.

66. Hare RD (1983). Diagnosis of antisocial personality disorder in two prison populations. Am J Psychiatry. 1983; 140:887-90.

67. Hare RD. Psychopathy and antisocial personality disorder: A case of diagnostic confusion. Psychiatr Times. 1996;13:39-40.

68. Hare RD, Hart SD. Commentary on antisocial personality disorder: The DSM-IV field trial. En: Livesley WJ, editor. The DSM-IV personality disorders. New York: Guilfod Press; 1995. p. 127-34.

69. Hare RD, Hart SD, Harpur TJ. Psychopathy and the DSM-IV criteria for antisocial personality disorder. J Abnorm Psychol. 1991;100: 391-98.

70. Hart SD, Hare RD. Psychopathy and antisocial personality disorder. Curr Opin on Psychiatry. 1996;9:129-32. 